\title{
Transversals in hypergraphs through a new combinatorial game
}

\author{
Wilder P. Mendes, ${ }^{(1)}$ Simone Dantas, ${ }^{(\mathbb{D}}$ Sylvain Gravier ${ }^{(i)}$
}

\begin{abstract}
In this paper, we explore a new combinatorial game called $(a, b)$ monochromatic transversal game proposed by Mendes et al. in 2020 . This game is defined over a hypergraph and it is played by two players, Alice and Bob, that alternately take turns colouring $a$ vertices in red, if the player is Alice, or $b$ vertices in blue, if the player is Bob. Alice wins the game if she obtains a red hyperedge transversal while Bob wins the game if he obtains a monochromatic blue hyperedge. Moreover, as part of the rules of this game, both players can start. In the next pages, we study the game over biclique-hypergraphs of powers of paths and of powers of cycles and show winning strategies for each player, according to the choices of $a$ and $b$.
\end{abstract}

\section{Introduction}

The graphs considered in this paper are undirected and simple. A clique of a graph $G$ is a subset of vertices of this graph, which induces a com-

2000 AMS Subject Classification: 05C57, 05C15, 05C65

Keywords and Phrases: cliques, bicliques, combinatorial games, hypergraphs, transversal, powers of paths, powers of cycles

This study was financed in part by the Coordenação de Aperfeiçoamento de Pessoal de Nível Superior - Brasil (CAPES) - Finance Code 001, CAPES-PrInt project number 88881.310248/2018-01, CNPq and FAPERJ. 
plete graph. Moreover, maximal clique is a clique that it is not properly contained in any other clique. A generalization of the definition of graphs is the concept of hypergraphs, where the edges, named hyperedges, have more than two incident vertices.

A clique-hypergraph of a graph $G$ is a hypergraph with the same vertex set of $G$ and whose hyperedges are the maximal cliques of $G$. This structure was firstly presented in 1991 by Duffus et al. [9] and was extensively studied later, for example in [1, 6, 10]. Further problems, that were initially stated for cliques, have their versions for bicliques [7, 8, 16].

A biclique of a graph $G$ is a subset of the vertex set of $G$ that induces a complete bipartite subgraph with at least one edge. So, a bicliquehypergraph of a graph $G$ is the hypergraph in which the vertex set is the same of $G$ and the hyperedge set is the set of all maximal bicliques of G. In 2014 Groshaus et al. [11] introduced and study biclique-colouring problem from the point of view of the computational complexity of the star and biclique coloring and choosability problems. After that, in 2015, Macêdo et al. [12] studied this problem over the classes of powers of paths and of powers of cycles.

By the point of view of combinatorial games [3, i.e., alternating finite two-player games of pure strategy in which all the relevant information is public to both players, as well as no randomness or luck are allowed, Butjás et al. 4, 5] presented a combinatorial game using the concept of tranversals in hypergraphs that was called transversal game. A transversal in a hypergraph is a subset of the vertex set that intersects every hyperedge [2]. From another perspective, in 2020, Mendes et al. [13] introduced a new Combinatorial game called the monochromatic transversal game.

In this game, Alice and Bob alternately colour one vertex of a hypergraph. Alice, who colours the vertices in red, wins the game if she obtains a red hyperedge transversal, while Bob wins it if he obtains a monochromatic blue hyperedge. We remark that both players can start the game. In [13], the authors analyzed the game played on clique-hypergraphs of complete graphs, paths, and powers of cycles where, for each of these 
graphs, they showed a strategy that allows one of the players to win the game.

In this paper, we study the $(a, b)$-monochromatic transversal game that was introduced by Mendes et al. 14] allowing, on each turn, Alice and Bob colour $a \geq 1$ and $b \geq 1$ vertices for $a, b \in \mathbb{N}^{*}$, respectively.

Following the idea that some problems studied for cliques were also considered over the perspective of bicliques, here we focus our attention on the game played over biclique-hypergraphs of powers of paths and powers of cycles, showing strategies related to the parameters $a$ and $b$ that allow each of the players to win the game.

We organize the paper as follows. We present the rules of the game, basic definitions, notation, and general properties in Section 2, In Section 3 , we analyze the $(a, b)$-monochromatic transversal game on bicliquehypergraphs of power of paths and of power of cycles, first for the cases where $a=b=1$, and later for $a, b \geq 1$. Finally, we present our conclusions in Section 4

\section{Basic definitions and general properties}

A hypergraph $\mathcal{H}$ is a pair $(\mathcal{V}, \mathcal{E})$ where $\mathcal{V}$ is a non-empty finite vertex set and $\mathcal{E}$ is a family of non-empty subsets of $\mathcal{V}$ called hyperedges.

The (a,b)-monochromatic transversal game, called $(a, b)-M T G$, is an avoider-enforcer game where two players, Alice and Bob, alternately colour the vertices of a hypergraph $\mathcal{H}$. At each turn, either Alice colours $a$ vertices in red or Bob colours $b$ vertices in blue. Alice wins the game if she obtains a red hyperedge transversal, that is, a subset of vertices of $\mathcal{V}$ that has a non-empty intersection with every hyperedge of $\mathcal{H}$. Bob wins the game if he obtains a monochromatic blue hyperedge of $\mathcal{E}$. We consider $a$ less than the size of a minimum transversal (resp. $b$ less than the size of the smallest hyperedge of $\mathcal{H}$ ), otherwise the game is trivial because Alice (resp. Bob) wins in her (resp. his) first turn. 
The game presents the following properties stated by Mendes et al. [14]:

Remark 2.1. 14] If there exists a strategy that allows Alice (resp. Bob) to win when Bob (resp. Alice) starts the $(a, b)$-MTG on a given hypergraph, then there exists a strategy that allows Alice (resp. Bob) to win when she (resp. he) starts the game on that hypergraph.

Remark 2.2. 14] If there exists a strategy that allows Alice (resp. Bob) to win the $\left(a_{0}, b_{0}\right)$-MTG played on a given hypergraph, independently of who starts it, then for any $a>a_{0}$ (resp. $b>b_{0}$ ) there exists a strategy that allows Alice (resp. Bob) to win the $\left(a, b_{0}\right)$-MTG (resp. $\left.\left(a_{0}, b\right)-\mathrm{MTG}\right)$ on that hypergraph, independently of who starts it.

The biclique-hypergraph, $\mathcal{H}_{\mathcal{B}}(G)=\left(V, \mathcal{E}_{\mathcal{B}}\right)$, of a graph $G=(V, E)$ is the hypergraph such that $V$ is the same vertex set of $G$ and the hyperedge set $\mathcal{E}_{\mathcal{B}}$ is the set of all maximal bicliques of $G$, that is, $\mathcal{E}_{\mathcal{B}}$ is the set of all maximal subsets of $V$ whose vertices induce a complete bipartite graph with at least one edge. Next, we consider biclique-hypergraphs of powers of paths and powers of cycles.

The $k$-th power of a path of order $n, P_{n}^{k}$, for $k \geq 1$, is a graph with $V\left(P_{n}^{k}\right)=\left\{v_{0}, v_{1}, \ldots, v_{n-1}\right\}$ and $\left\{v_{i}, v_{j}\right\} \in E\left(P_{n}^{k}\right)$ if and only if $|i-j| \leq k$. Note that, $P_{n}^{1} \simeq P_{n}$ and $P_{n}^{k} \simeq K_{n}$ for $n \leq k+1$. For $n \in\{2,3\}$ a $P_{n}$ maximal biclique is a maximal biclique of the graph which is isomorphic to a path $P_{n}$.

A $k$-th power of cycle of order $n, C_{n}^{k}$, for $k \geq 1$, is a graph on $n$ vertices whose vertex set is $V\left(C_{n}^{k}\right)=\left\{v_{i}: i \in \mathbb{Z}_{n}\right\}$ and whose edges $\left\{v_{i}, v_{j}\right\}, i, j \in$ $\mathbb{Z}_{n}$ have the property that $i=j \pm r(\bmod n)$ for some $r \in\{1,2, \ldots, k\}$. In what follows, the operations are made module n. Observe that $C_{n}^{k} \simeq K_{n}$ for $k \geq\left\lfloor\frac{n}{2}\right\rfloor$. A $C_{4}$ maximal biclique is a maximal biclique of the graph which is isomorphic to a cycle $C_{4}$. A non-complete power of a path $P_{n}^{k}$ (resp. cycle $C_{n}^{k}$ ) is such that $P_{n}^{k} \not K_{n}$ (resp. $C_{n}^{k} \not K_{n}$ ). A universal vertex is a vertex of $G$ that is adjacent to all other vertices of the $G$.

Recall that we consider only maximal bicliques to see that the following holds. 
Lemma 2.1. [12] Every $P_{2}$ maximal biclique of a power of path or of a power of a cycle is a pair of universal vertices.

As consequence of Lemma 2.1, a non-complete power of a cycle has no $P_{2}$ maximal bicliques, as well as a power of a path $P_{n}^{k}$ with $n \geq$ $2 k+1$. Considering that powers of cycles and powers of paths are $K_{1,3}$ free, and that powers of paths are $C_{4}$-free, Macêdo Filho et al. [12] show the following results:

Theorem 2.1. [12] Let $P_{n}^{k}$ be a non-complete power of a path and let $C_{n}^{k}$ be a non-complete power of a cycle.

(i) the maximal bicliques of $P_{n}^{k}$ are precisely: $P_{2}$ maximal bicliques and $P_{3}$ maximal bicliques, if $k+2 \leq n \leq 2 k$; and $P_{3}$ maximal bicliques, if $n \geq 2 k+1$.

(ii) the maximal bicliques of $C_{n}^{k}$ are precisely: $C_{4}$ maximal bicliques, if $2(k+1) \leq n \leq 3 k+1 ; P_{3}$ maximal bicliques and $C_{4}$ maximal bicliques, if $3 k+2 \leq n \leq 4 k$; and $P_{3}$ maximal bicliques if $n \geq 4 k+1$.

Theorem 2.1 also implies the next result that characterizes the $P_{2}$ maximal bicliques for a given power of a path:

Lemma 2.2. If $P_{n}^{k}$ is a power of a path with $k+2 \leq n \leq 2 k$ and $k \geq 2$, then the $P_{2}$ maximal bicliques of $P_{n}^{k}$ are given by the $\left(\begin{array}{c}|S| \\ 2\end{array}\right)$ edges of the complete graph with $|S|$ vertices where $S=\left\{v_{(n-1)-k}, v_{n-k}, \ldots, v_{k}\right\}$.

\section{The $(a, b)$-monochromatic transversal game}

In [15], the authors showed the following results for the $(1,1)$-MTG: Alice wins the game played on $\mathcal{H}_{\mathcal{B}}\left(P_{4}^{2}\right)$ and on $\mathcal{H}_{\mathcal{B}}\left(P_{5}^{2}\right)$, independently of who starts playing; the player who has the first turn wins the game played on $\mathcal{H}_{\mathcal{B}}\left(P_{n}^{3}\right), 5 \leq n \leq 6$ and on $\mathcal{H}_{\mathcal{B}}\left(P_{n}^{2}\right), 6 \leq n \leq 7$; and finally Bob wins the game played on $\mathcal{H}_{\mathcal{B}}\left(P_{n}^{k}\right), k \geq 4$ and $k+2 \leq n \leq 2 k$ and on $\mathcal{H}_{\mathcal{B}}\left(P_{n}^{k}\right)$, $k=2$ and $n \geq 8$, or $k \geq 3$ and $n \geq 2 k+1$, independently of who starts playing. 
Therefore, since Bob has a strong advantage in the (1,1)-MTG played on powers of paths, the next theorem summarizes the results of [15] for the case Bob starts playing.

Theorem 3.1. [15] If $P_{n}^{k}$ is a power of a path with $k \geq 3$ and $n \geq k+2$, then there exists a strategy that allows Bob to win the $(1,1)-M T G$ played on the biclique-hypergraph $\mathcal{H}_{\mathcal{B}}\left(P_{n}^{k}\right)$ when he starts playing.

As in the case of the powers of paths, by [15], Bob wins the $(1,1)-\mathrm{MTG}$ played on clique-hypergraphs of powers of cycles $\mathcal{H}\left(C_{n}^{k}\right)$ for $k \geq 2$ and $n \geq 2 k+3$, independently of who starts playing.

By the characterization of maximal bicliques given by Theorem 2.1, if Bob starts playing and $b \geq 4$ (resp. $b \geq 3$ ), then he wins the $(a, b)$-MTG on $\mathcal{H}_{\mathcal{B}}\left(C_{n}^{k}\right)$ since he makes a monochromatic blue $C_{4}$ (resp. $P_{3}$ ) maximal biclique on his first turn. Hence, we present next the study of the $(a, 2)$ MTG played on $\mathcal{H}_{\mathcal{B}}\left(C_{n}^{k}\right)$ for $n \geq 3 k+2$ and $k \geq 2$.

Theorem 3.2. If $C_{n}^{k}$ is a power of a cycle with $3 k+2 \leq n \leq 4 k+2$ and $k \geq 2$, then Alice wins the (a,2)-MTG played on the biclique-hypergraph $\mathcal{H}_{\mathcal{B}}\left(C_{n}^{k}\right)$ if $a \geq 2 k$.

Proof. Suppose that Bob starts playing the $(a, 2)-$ MTG on $\mathcal{H}_{\mathcal{B}}\left(C_{n}^{k}\right)$ and $a \geq 2 k$ for $k \geq 2$. Assume that Bob colours blue $v_{0}$ and $v_{i}$ on his first turn. We define $\mathcal{A}$ as the subset of vertices coloured by Alice after Bob's first turn. If $v_{0}$ and $v_{i}$ are adjacent then, by symmetry, one may assume that $1 \leq i \leq k$. So, Alice colours red all the vertices in $\mathcal{A}=\left\{v_{k+1}, \ldots, v_{2 k}\right\} \cup$ $\left\{v_{n-k}, \ldots, v_{n-1}\right\}$. Since $n \leq 4 k+2$, the graph $G$ induced by $V\left(C_{n}^{k}\right) \backslash \mathcal{A}$ is composed of the disjoint union of at most two cliques, say $\left\{v_{0}, v_{1}, \ldots, v_{k}\right\}$ and $\left\{v_{2 k+1}, \ldots v_{n-k-1}\right\}$ (no edges between them). Therefore, Bob cannot colour blue a $P_{3}$ maximal biclique in $G$ and so Alice wins.

Finally, assume that $v_{0}$ and $v_{i}$ are non-adjacent and, by the symmetry of $C_{n}^{k}$, without loss of generality we assume that $k+1 \leq i \leq\left\lfloor\frac{n}{2}\right\rfloor$. Alice colours red all the vertices in $\mathcal{A}=\left\{v_{1}, \ldots, v_{k}\right\} \cup\left\{v_{\left\lfloor\frac{n}{2}\right\rfloor+1}, \ldots, v_{\left\lfloor\frac{n}{2}\right\rfloor+k}\right\}$. We note that, $n-\left\lfloor\frac{n}{2}\right\rfloor-k \leq k+1$. Again, the graph $G$ induced by $V\left(C_{n}^{k}\right) \backslash$ 
$\mathcal{A}$ is the disjoint union of at most two cliques, say $\left\{v_{k+1}, \ldots v_{\left\lfloor\frac{n}{2}\right\rfloor}\right\}$ and $\left\{v_{\left\lfloor\frac{n}{2}\right\rfloor+k+1}, \ldots, v_{0}\right\}$. Therefore, as previously Alice wins. By Remark 2.1. Alice wins the $(a, 2)$-MTG played on $\mathcal{H}_{\mathcal{B}}\left(C_{n}^{k}\right)$ if $a \geq 2 k$.

Theorem 3.3. If $C_{n}^{k}$ is a power of a cycle with $5 k+2 \leq n \leq 6 k+2$ and $k \geq 2$, then Alice wins the (a,2)-MTG played on the biclique-hypergraph $\mathcal{H}_{\mathcal{B}}\left(C_{n}^{k}\right)$ if $a \geq 3 k$.

Proof. Suppose that Bob starts playing the $(a, 2)-\mathrm{MTG}$ on $\mathcal{H}_{\mathcal{B}}\left(C_{n}^{k}\right)$ and $a \geq 3 k$ for $k \geq 2$. Assume that Bob colours blue $v_{0}$ and $v_{i}$ on his first turn. We define $\mathcal{A}$ as the subset of vertices coloured by Alice after Bob's first turn. If $v_{0}$ and $v_{i}$ are adjacent then, by symmetry, one may assume that $1 \leq i \leq k$. Let $\mathcal{A}=\left\{v_{k+1}, \ldots, v_{2 k}\right\} \cup\left\{v_{n-3 k-1}, \ldots, v_{n-2 k-2}\right\} \cup$ $\left\{v_{n-k}, \ldots, v_{n-1}\right\}$. Since $n \leq 6 k+2$ the subgraph $G$ induced by $V\left(C_{n}^{k}\right) \backslash \mathcal{A}$ is composed of disjoint cliques. Therefore, Alice wins preventing Bob of colouring a $P_{3}$ maximal biclique in $G$.

Now, if $v_{0}$ and $v_{i}$ are non-adjacent then, by the symmetry of $C_{n}^{k}$, without loss of generality we assume that $k+1 \leq i \leq\left\lfloor\frac{n}{2}\right\rfloor$. If $k+1 \leq i \leq 2 k$ then $\mathcal{A}=\left\{v_{1}, \ldots, v_{k}\right\} \cup\left\{v_{2 k+1}, \ldots, v_{3 k}\right\} \cup\left\{v_{n-2 k}, \ldots, v_{n-k-1}\right\}$; else if $2 k+1 \leq i \leq\left\lfloor\frac{n}{2}\right\rfloor$ then $\mathcal{A}=\left\{v_{k+1}, \ldots, v_{2 k}\right\} \cup\left\{v_{\left\lfloor\frac{n}{2}\right\rfloor+1}, \ldots, v_{\left\lfloor\frac{n}{2}\right\rfloor+k}\right\} \cup$ $\left\{v_{n-k}, \ldots, v_{n-1}\right\}$. We note that, $\left\lfloor\frac{n}{2}\right\rfloor-2 k \leq k+1$. So, in both cases, the subgraph $G$ induced by $V\left(C_{n}^{k}\right) \backslash \mathcal{A}$ is composed of disjoint cliques and Alice wins. Therefore, by Remark 2.1. Alice wins the $(a, 2)-\mathrm{MTG}$ played on $\mathcal{H}_{\mathcal{B}}\left(C_{n}^{k}\right)$ if $a \geq 3 k$.

The idea of the proofs of the previous results can be extended to the next two theorems but, in these cases, the vertices coloured by Alice depend on the parameter $\epsilon$.

Theorem 3.4. If $C_{n}^{k}$ is a power of a cycle with $4 k+2 \leq n \leq 5 k+2-\epsilon$ and $k \geq 2$, then Alice wins the (a,2)-MTG played on the biclique-hypergraph $\mathcal{H}_{\mathcal{B}}\left(C_{n}^{k}\right)$, if $a \geq 3 k-\epsilon$ for $0 \leq \epsilon \leq k$. 
Theorem 3.5. If $C_{n}^{k}$ is a power of a cycle with $6 k+2<n \leq 8 k+2-\epsilon$ and $k \geq 2$, then Alice wins the (a,2)-MTG played on the biclique-hypergraph $\mathcal{H}_{\mathcal{B}}\left(C_{n}^{k}\right)$, if $a \geq 4 k-\left\lfloor\frac{\epsilon}{2}\right\rfloor$ for $0 \leq \epsilon \leq 2 k$.

\section{Conclusion}

In [15], it is shown that for the $(1,1)-\mathrm{MTG}$ played on the bicliquehypergraphs of $P_{n}^{k}$, where Bob has a major advantage always winning whenever he starts the game for $k \geq 3$ and $n \geq k+2$, as well as, by Theorem 3.1 and Remark 2.2, we see that when $b \geq 2$ Bob also always wins the $(1, b)$-MTG played for the same values of $n$ and $k$.

Knowing the behavior of the $(1,1)-\mathrm{MTG}$ over $\mathcal{H}_{\mathcal{B}}\left(P_{n}^{k}\right)$ and $\mathcal{H}_{\mathcal{B}}\left(C_{n}^{k}\right)$, we establish for $3 k+2 \leq n \leq 8 k+2$ the $(a, 2)$-MTG played on $\mathcal{H}_{\mathcal{B}}\left(C_{n}^{k}\right)$, $k \geq 2$, and so we summarize our results of Section 3 on Table 1 , considering $0 \leq \epsilon \leq k$ for Theorem 3.4 and $0 \leq \epsilon \leq 2 k$ for Theorem 3.5. The last three columns show the winner when Alice (resp. Bob) starts playing and the corresponding result in this paper.

Moreover, Theorems 3.2 to 3.5 give us an idea of a lower bound for $a$ when $n \geq 8 k+2$ and $k \geq 2$, that is, $a \geq 4 k$ to guarantee Alice's winning strategy.

\begin{tabular}{|c|c|c|c|c|}
\hline Value for $n$ & Value for $a$ & Alice & Bob & Reference \\
\hline \hline $3 k+2 \leq n \leq 4 k+2$ & $a \geq 2 k$ & A & A & Theorem 3.2 \\
\hline $4 k+2 \leq n \leq 5 k+2-\epsilon$ & $a \geq 3 k-\epsilon$ & A & A & Theorem 3.4 \\
\hline $5 k+2 \leq n \leq 6 k+2$ & $a \geq 3 k$ & A & A & Theorem 3.3 \\
\hline $6 k+2 \leq n \leq 8 k+2-\epsilon$ & $a \geq 4 k-\left\lfloor\frac{\epsilon}{2}\right\rfloor$ & A & A & Theorem 3.5 \\
\hline
\end{tabular}

Table 1: Results for $C_{n}^{k}$ and winners A (Alice) and B (Bob) 


\section{References}

[1] G. Bacsó, S. Gravier, A. Gyárfás, M. Preissmann, and A. Sebő, Coloring the maximal cliques of graphs, SIAM J. Discrete Math. 17 (2014) 361-376.

[2] C. Berge, "Hypergraphs: Combinatorics of finite sets", first Ed., North-Holland, Amsterdam, 1989.

[3] E.R. Berlekamp, J.H. Conway, and R.K. Guy, "Winning Ways for Your Mathematical Plays: Volume 1 and 2", first Ed., A. K. Peters Press, Natick, 1981.

[4] C. Bujtás, M.A. Henning, and Z. Tuza, Transversal game on hypergraphs and the $\frac{3}{4}$-conjecture on the total domination game, SIAM J. Discrete Math. 30 (2016) 1830-1847.

[5] C. Bujtás, M.A. Henning, and Z. Tuza, Bounds on the game transversal number in hypergraphs, Eur. J. Combin. 59 (2017) 34-50.

[6] C.N. Campos, S. Dantas, and C.P. de Mello, Colouring cliquehypergraphs of circulant graphs, Graphs Combin. 29 (2013) 17131720.

[7] D. Conlon, On-line Ramsey numbers, SIAM J. Discrete Math. 23 (2009/2010) 1954-1963.

[8] V.M.F. Dias, C.M.H. de Figueiredo, and J.L. Szwarcfiter, On the generation of bicliques of a graph, Discrete Appl. Math. 155 (2007) $1826-1832$.

[9] D. Duffus, B. Sands, N. Sauer, and R.E. Woodrow, Two-colouring all two-element maximal antichains, J. Combin. Theory Ser. A 57 (1991) 109-116.

[10] S. Gravier, C.T. Hoàng, and F. Maffray, Coloring the hypergraph of maximal cliques of a graph with no long path, Discrete Math. 272 (2003) 285-290. 
[11] M. Groshaus, F.J. Soulignac, P. Terlisky, The star and biclique coloring and choosability problems, Journal of Graph Algorithms and Applications 18 (2014) 347-383.

[12] H.B. Macêdo Filho, S. Dantas, R.C.S. Machado, and C.M.H. Figueiredo, Biclique-colouring verification complexity and biclique-colouring power of paths, Discrete Appl. Math. 192 (2015) 65-76.

[13] W.P. Mendes, S. Dantas, S. Gravier, and R. Marinho, The monochromatic transversal game on clique-hypergraphs of powers of cycles, In Proceedings of 18th Cologne-Twente Workshop on Graphs and Combinatorial Optimization (CTW 2020).

[14] W. P. Mendes, S. Dantas, S. Gravier, and R. Marinho, The $(a, b)$ monochromatic transversal game on clique-hypergraphs of powers of cycles. Submitted to Discrete Appl. Math.

[15] W. P. Mendes, S. Dantas and S. Gravier, The (a,b)-monochromatic transversal game on biclique-hypergraphs of powers of paths and of powers of cycles. In: XI LAGOS. Latin-American Algorithms, Graphs and Optimization Symposium, 2021, São Paulo. Electronic Notes in Theoretical Computer Science, to appear.

[16] E. Prisner, Bicliques in graphs. I: Bounds on their number, Combinatorica 20 (2000) 109-117. 
Wilder P. Mendes

Niterói, Brazil

Fluminense Federal University

wmendes@id.uff.br

Sylvain Gravier

Saint-Martin-d'Hères, France CNRS, Univ. Grenoble Alpes

sylvain.gravier@ujf-grenoble.fr
Simone Dantas

Niterói, Brazil

Fluminense Federal University

sdantas@id.uff.br 